\title{
Emmprin, released as a microvesicle in epithelioid sarcoma, interacts with fibroblasts
}

\author{
MIKIKO AOKI $^{1}$, KAORI KOGA ${ }^{1}$, MAKOTO HAMASAKI ${ }^{1}$, \\ NAGAYASU EGAWA ${ }^{2}$ and KAZUKI NABESHIMA ${ }^{1}$ \\ ${ }^{1}$ Department of Pathology, Fukuoka University Hospital and School of Medicine, Fukuoka, Japan; \\ ${ }^{2}$ Department of Pathology, University of Cambridge, Cambridge CB2 1QP, UK
}

Received February 10, 2017; Accepted March 20, 2017

DOI: 10.3892/ijo.2017.3986

\begin{abstract}
Emmprin (extracellular matrix metalloproteinase inducer, CD147) is a glycosylated transmembrane protein, consisting of two immunoglobulin domains, that stimulates the production of matrix metalloproteinases (MMPs) by tumor-associated fibroblasts. These effects play important roles in tumor invasion and metastasis. However, the precise mechanisms by which emmprin acts on fibroblasts have not been fully elucidated, especially in sarcoma cells. Previously, we demonstrated that emmprin, expressed in conditioned medium collected from the epithelioid sarcoma cell line (FU-EPS-1), stimulates MMP-2 production via interactions with fibroblasts. In this study, we used microvesicles derived from sarcoma cells, and determined whether emmprin exists in the microvesicles, which enhance the production of MMP-2 via fibroblasts. Microvesicles released from FU-EPS-1 cells were shown to contain full-length emmprin, identified as a $45-\mathrm{kDa}$ protein characterized by polylactosamine glycosylation. Microvesicles collected from FU-EPS-1 cells transfected with emmprin-specific siRNA or transduced with shRNA displayed significantly reduced MMP-2 production by fibroblasts compared with those from control-transfected cells. Our findings show that emmprin is released through microvesicle shedding in sarcoma cells, and emmprin in microvesicles regulates MMP-2 production by influencing the activity of fibroblasts located at sites distant from the tumor cells.
\end{abstract}

Correspondence to: Dr Kazuki Nabeshima, Department of Pathology, Fukuoka University School of Medicine, 7-45-1 Nanakuma, Jonan-ku, Fukuoka 814-0180, Japan

E-mail:kaznabes@fukuoka-u.ac.jp

Abbreviations: emmprin, extracellular matrix metalloproteinase inducer, CD147; MMP, matrix metalloproteinase; MV, microvesicle; siRNA, small interference RNA; shRNA, short hairpin RNA; FCS, fetal calf serum

Key words: sarcoma, microvesicles, emmprin, MMP-2, fibroblasts, tumor invasion, tumor metastasis

\section{Introduction}

Tumor cells and stromal fibroblasts mutually interact. Particularly, a process of degradation and reconstruction of the neighboring stroma is essential for cancer invasion. Matrix metalloproteinases (MMPs) play a central role in this process. Emmprin regulates the production of MMP-2 by mediating this interaction between the tumor cells and the fibroblasts. Emmprin is a membrane glycoprotein with two extracellular immunoglobulin-like domains and has molecular weight of 44-66 kDa depending on the degree of glycosylation $(1,2)$. Emmprin is expressed more abundantly in tumor cells than in fibroblasts. A number of studies have reported that emmprin is expressed at a significantly higher level in malignant tumors compared to benign or normal tissues, and that the high emmprin expression is correlated with malignancy and/or poor prognosis $(3,4)$. In humans, 24 MMPs that degrade stroma have been discovered. They are broadly classified into secretory and membrane types. MMP-2 represents the secretory MMPs in tumors. MMP-2 is expressed in both tumor cells and fibroblasts, with predominant expression observed in fibroblasts in direct contact with tumor cells. However, not only the fibroblasts neighboring the tumor cells but also those located at sites distant from tumor cells express MMP-2.

Co-culture is one of the methods of observing interactions between the tumor cells and the fibroblasts. Co-culture of fibroblasts and tumor cells significantly enhances the MMP-2 expression by fibroblasts via emmprin. This phenomenon is also observed when an epithelioid sarcoma cell line is used (5). Our laboratory previously demonstrated that conditioned medium of epithelioid sarcoma cells contains full-length emmprin, and that the addition of this conditioned medium to fibroblasts led to enhanced MMP-2 expression by the fibroblasts, which was suppressed by anti-emmprin neutralizing antibody (5). This result indicated that emmprin is released from the sarcoma cells and acts on fibroblasts located at sites distant from the tumor cells.

Emmprin is likely secreted in two forms: released by proteolytic cleavage, or released with the membrane in the form of microvesicles. Egawa et al have reported the proteolytic cleavage of emmprin by MT1-MMP based on their experiments using human epidermoid carcinoma cell line and human fibrosarcoma cell line (6). In our prior experiments 
using sarcoma cells, emmprin in the conditioned medium was detected as a full length protein. However, the precise mechanism, by which full length emmprin is released, have not been determined.

In the present study, we have used microvesicles to directly examine our phenomenon-based hypothesis that emmprin exists in the form of microvesicles in the conditioned medium, and that emmprin packaged in the microvesicles facilitates MMP-2 production by acting on fibroblasts located at distant sites from the tumor cells.

\section{Materials and methods}

Cell culture. Epithelioid sarcoma cell line FU-EPS-1 was established in our laboratory from a patient with epithelioid sarcoma who had not received any chemotherapy before surgical resection (7). The cell line was maintained in growth medium, D-MEM/Ham's F-12 (Wako, Japan), supplemented with $10 \%$ fetal calf serum (FCS). The human dermal fibroblast ST353 was obtained from non-lesional dermis around nodular fascitis. ST353i was immortalized by transduction of human telomerase reverse transcriptase (hTERT).

Preparation of microvesicles. Microvesicles from cell-cultured medium were prepared as previously described (8-10). FU-EPS-1 cells were cultured to sub-confluency in the growth medium. The cells were washed with serum-free medium and cultured further in DMEM/Ham's-F12 without serum for $48 \mathrm{~h}$. Conditioned medium was centrifuged at $1500 \mathrm{~g} 15 \mathrm{~min}$ to remove cells and large debris. Supernatant was centrifuged at 50,000 $\mathrm{g}$ for $1 \mathrm{~h}$ at $4^{\circ} \mathrm{C}$. Pelleted microvesicles were re-suspended in serum-free medium containing $0.2 \%$ lactalbumin enzymatic hydrolysate. Vesicles were quantified based on tumor cell counts.

Electron microscopy. Microvesicles were applied on 400 mesh nickel parlodion-coated grids and allowed to settle. Samples were blocked in 5\% normal goat serum for $30 \mathrm{~min}$, rinsed in PBS and incubated with anti-emmprin antibody at $4{ }^{\circ} \mathrm{C}$ overnight. Grids were washed with PBS, incubated with gold nanoparticle conjugated anti-mouse secondary antibody (Abcam, Cambridge, UK) for $1 \mathrm{~h}$ and then rinsed with PBS again. Grids were fixed in $2 \%$ glutaraldehyde in PBS, rinsed, and then negatively stained with $1 \%$ phosphotungstic acid (pH 6.0), before being analyzed under an electron microscope. Specificity of immunolabeling was determined by comparing results obtained with gold-labeled secondary antibody only.

Immunoblotting. SDS-PAGE and immunoblotting of microvesicles and cell lysates were performed using 4-15\% Mini-PROTEAN TGX gel (Bio-Rad, Hercules, CA, USA), immobilon membrane (Millipore, Bedford, MA, USA), antibodies against emmprin (monoclonal antibody, clone 109403, R\&D System, Flanders, NJ, USA; goat polyclonal antibody against $\mathrm{N}$ - and C-terminal region of emmprin, Santa Cruz Biotechnology, Santa Cruz, CA, USA), and MMP-2 monoclonal antibody (Daiichi Fine Chemical, Toyama, Japan) as described previously (5).

Glycosidase digestions. Extracted membrane protein from microvesicles were reacted with incubation buffer $(100 \mathrm{mM}$
$\mathrm{NaH}_{2} \mathrm{PO}_{4}(\mathrm{pH} 7.0), 10 \mathrm{mM}$ EDTA, $1 \%$ NP-40, 0.1\% SDS, $1 \%$ 2-mercaptoethanol) containing $10 \mathrm{U} / \mathrm{ml} \mathrm{N}$-glycosidase $\mathrm{F}$ (Roche, Basel, Switzerland) for $24 \mathrm{~h}$ at $37^{\circ} \mathrm{C}$. The trichloroacetic acid was added to a final concentration of $3.3 \%$, and the samples were incubated at $4^{\circ} \mathrm{C}$ overnight. The samples were centrifuged at $10,000 \mathrm{rpm}$ for $10 \mathrm{~min}$ to remove supernatant. The pellet was lysed in $4 \mathrm{X}$ concentrated Laemmli sample buffer and analyzed by SDS/PAGE and immunoblotting.

Precipitation with biotinylated Sophora japonica agglutinin. Microvesicles were lysed in precipitation buffer (1\% CHAPS, $25 \mathrm{mM}$ HEPES, pH 7.5, $150 \mathrm{mM} \mathrm{NaCl}, 5 \mathrm{mM} \mathrm{MgCI}_{2}$, protease inhibitor cocktail) at $4^{\circ} \mathrm{C}$ overnight. After centrifugation $(42,000 \mathrm{rpm}, 1 \mathrm{~h})$, the supernatant was incubated at $4^{\circ} \mathrm{C}$ overnight with biotinylated Sophora japonica agglutinin (PHA-L, Vector Laboratories, Burlingame, CA, USA), followed by incubation with avidin agarose beads (Thermo Fisher Scientific, Waltham, MA, USA) at $4^{\circ} \mathrm{C}$ for $1 \mathrm{~h}$. After washing with precipitation buffer and centrifugation $(4,000 \mathrm{rpm}, 1 \mathrm{~min})$, the pellet was lysed in $4 \mathrm{X}$ concentrated Laemmli sample buffer and analyzed by SDS/PAGE and immunoblotting.

Transient knockdown of emmprin by RNA interference. Small interference RNAs (siRNAs) sequences were used for transient knockdown of emmprin mRNA. siRNAs targeting the emmprin were designed and synthesized (Invitrogen Corp., Carlsbad, CA, USA), and transfection was carried out using Oligofectamine transfection reagent in Opti-MEM (Thermo Fisher Scientific) in the absence of serum and antibiotics according to the manufacturer's instructions. Knockdown of emmprin expression in FU-EPS-1 cells was analyzed by immunoblotting.

Stable knockdown of emmprin by RNA interference. To establish the cells stably expressing shRNA against emmprin, we used the BLOCK-iT Lentiviral RNAi Expression system (Invitrogen Corp.), as described previously $(11,12)$. Genespecific insert (5'-CACAGTCTTCACTACCGTAGCGAACT ACGGTAGTGAAGACTGTGC-3') was cloned into pLenti6 according to the manufacturer's instructions. The lentivirus was produced in HEK 293FT cells using the ViraPower lentiviral expression system (Invitrogen), and the virus-containing media were harvested for infection. Lentivirus expressing LacZ shRNA was used as a control. Stable shRNA expressing cells were propagated and maintained in the presence of blasticidin ( $5 \mu \mathrm{g} / \mathrm{ml}$; Invitrogen).

Enzyme-linked immunosorbent assay (ELISA). The protein concentrations of MMP-2 were measured using human MMP-2 Quantikine ELISA kit (R\&D System, Minneapolis, $\mathrm{MN}, \mathrm{USA})$ as per the manufacturer's instructions.

\section{Results}

Determination of emmprin localization in microvesicles using immunoelectron micrographs. We first examined whether emmprin protein exists in microvesicles using immunoelectron microscopy. Microvesicles purified by ultracentrifugation obtained from the conditioned medium of the FU-EPS-1 cells were subjected to immunoelectron micrography using post- 


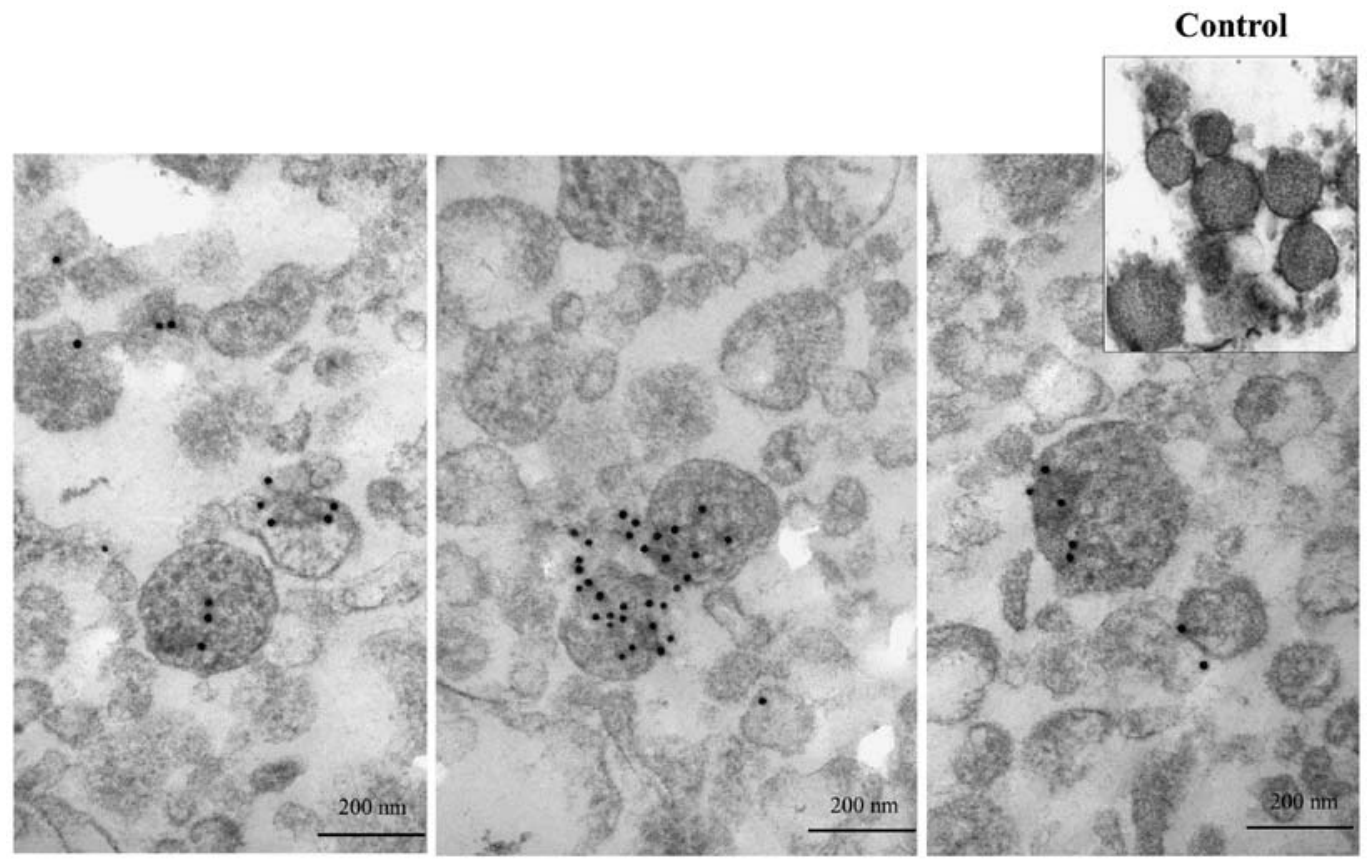

Figure 1. Emmprin exists in microvesicles derived from FU-EPS-1 cells. Electron microscopically, 100-200 nm sized microvesicles were observed in ultracentrifugal supernatant. Black spots indicate emmprin localization. No gold nanoparticles were noted in the control microvesicles.

\section{IB: Emmprin}

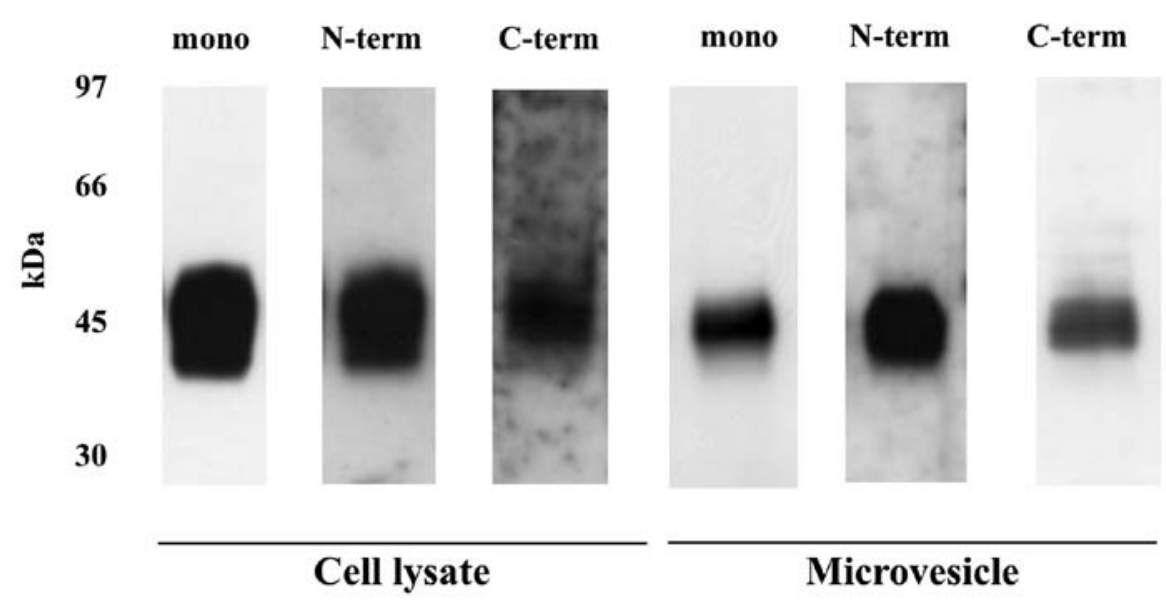

Figure 2. Microvesicles contain the full-length emmprin. Immunoblotting was performed using three types of anti-emmprin antibodies: monoclonal, N-term, and C-term. The bands obtained for the membrane protein extracts of total cell lysate and from the membrane protein extract of microvesicles are shown. Identical band $(\sim 45 \mathrm{kDa})$ was observed in membrane proteins from both total cell lysate and microvesicles.

embedding labeling methods. Microvesicles showed closed circle structures of 100-300 $\mathrm{nm}$ in diameter, similar to those of prior reports $(10,13)$. Black spots represent gold particles conjugated with anti-emmprin monoclonal antibody and indicate emmprin localization in microvesicles (Fig. 1). No gold particles were found in the control electron micrographs. Our data indicates the presence of emmprin in the microvesicles collected from FU-EPS-1 cells.

Full length emmprin expression in microvesicles released from epithelioid sarcoma cells. We next examined whether emmprin contained in the microvesicles was the full-length protein identical to the form expressed on the tumor cell surface. Membrane proteins were extracted from total cell lysates and microvesicles, both obtained from the FU-EPS-1 cells. Immunoblotting with anti-emmprin monoclonal antibody detected $\sim 45 \mathrm{kDa}$ emmprin band, which was also observed with antibodies raised against the $\mathrm{N}$ - and $\mathrm{C}$-terminal domain of emmprin, indicative of the release of full-length emmprin. Almost identical emmprin immunoreactivity was observed in total cell lysate and microvesicles (Fig. 2). These findings indicate that microvesicles also contain the full-length emmprin.

Presence of $N$-glycosylation with polylactosamine in emmprin released as microvesicles. We assessed the glycosylation 


\section{IB: Emmprin}

A

B

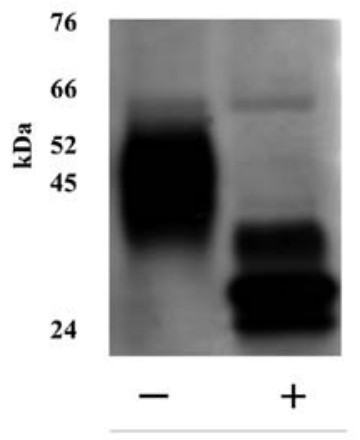

N-glycosidase $\mathbf{F}$

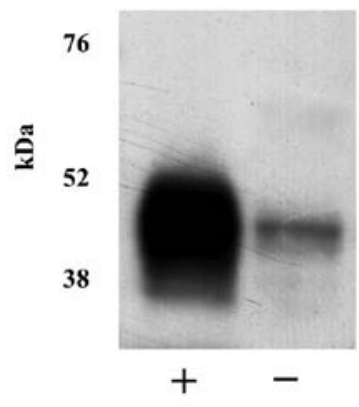

Precipitation using PHA-L

Figure 3. Evidence for N-linked glycosylation and presence of polylactosamines. (A) After treatment with $\mathrm{N}$-glycosidase F, the 45-kDa emmprin band derived from microvesicles was diminished and the 27-kDa core protein band was detected. (B) Emmprin derived from microvesicles precipitated with the lectin PHA-L (lane +).

of the emmprin released in the microvesicles. It is reported that emmprin-induced stimulation of MMP production in fibroblasts is dependent on N-glycosylation of its extracellular domains (14-16). Treatment of microvesicles with $\mathrm{N}$-glycosidase $\mathrm{F}$, which removes all glycosylation, increased the electrophoretic mobility of the observed emmprin protein band. A $27-\mathrm{kDa}$ band was observed upon treatment with $\mathrm{N}$-glycosidase $\mathrm{F}$, which is consistent with the size of the core emmprin protein (Fig. 3A). Further, emmprin released as microvesicles was precipitated by PHA-L lectin, indicative of the presence of $\beta 1,6$ (beta 1,6 ) branching and polylactosamines (Fig. 3B) (17,18). Overall, the data suggest that the full-length 45-kDa emmprin protein, identified in the microvesicles released from FU-EPS-1 cells, was characterized by polylactosamine glycosylation.

Emmprin derived from microvesicles regulates MMP-2 production by fibroblasts. Finally, we examined the function of emmprin in microvesicles. In particular, we were interested in elucidating whether emmprin in microvesicles also

A

IB

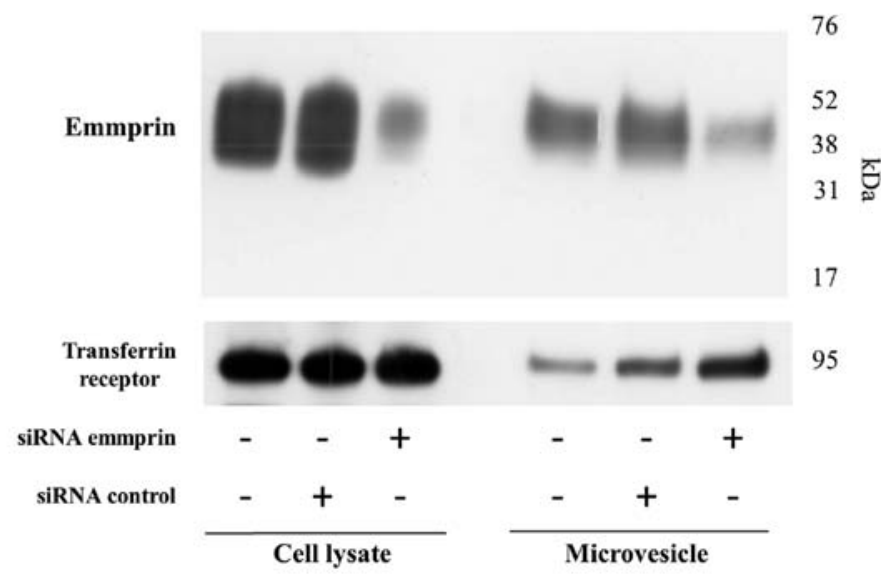

B

IB: MMP-2

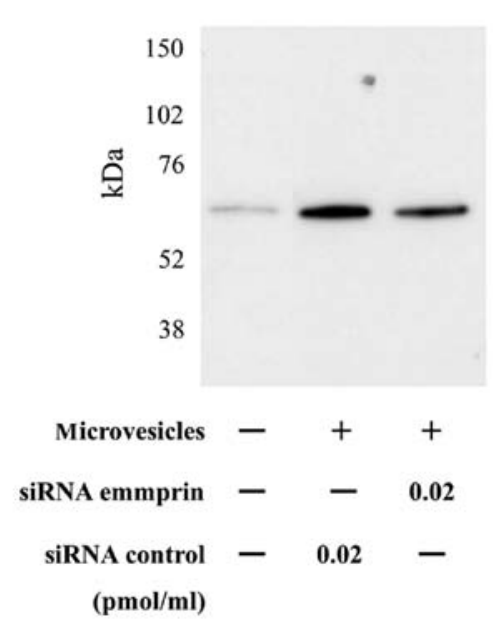

ELISA (MMP-2)

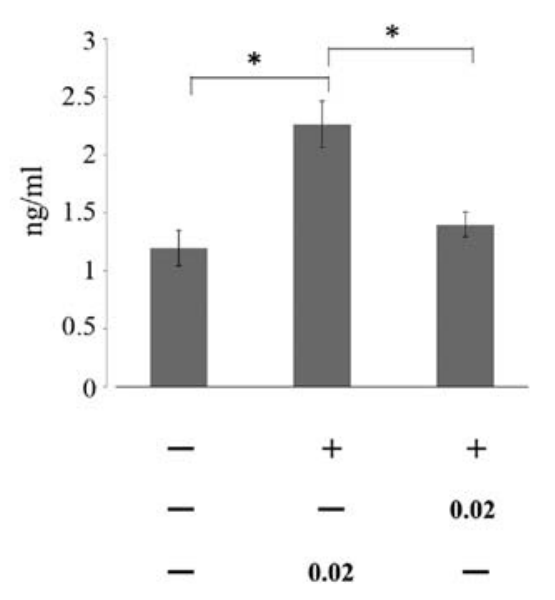

Figure 4. siRNA knockdown of emmprin in sarcoma cells and MMP-2 production from fibroblasts. (A) siRNA knockdown of emmprin reduced its expression in both total cell lysate and microvesicles. Compared to non-treatment (left lane) and control siRNA (middle lane), emmprin expression was inhibited by siRNA knockdown targeting emmprin (right lane). (B) Emmprin in microvesicles regulate MMP-2 production from fibroblasts. MMP-2 production by fibroblasts was significantly reduced when treated with microvesicles derived from sarcoma cells transiently transfected with emmprin specific siRNA compared to those from control siRNA transfection. These results were confirmed by immunoblot and ELISA $\left({ }^{*} \mathrm{p}<0.01\right)$. 
A

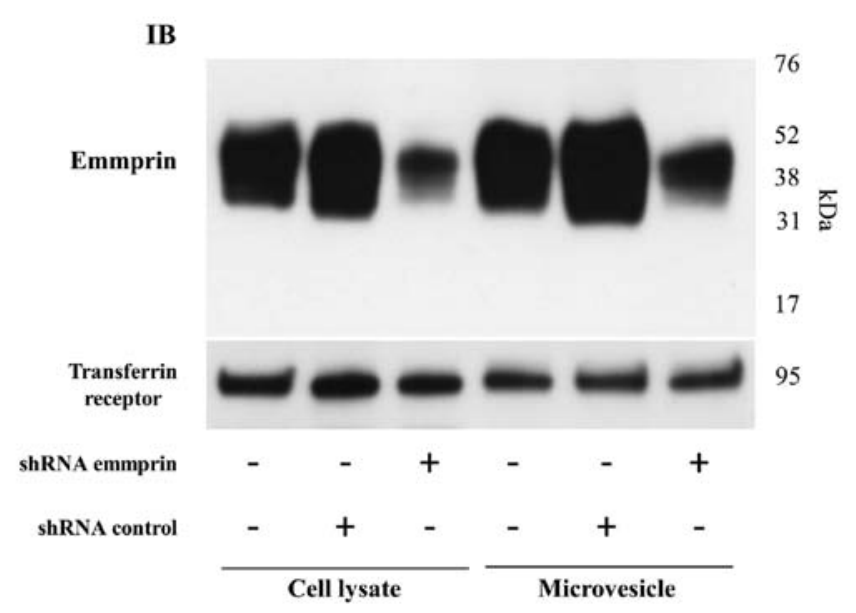

B

IB: MMP-2

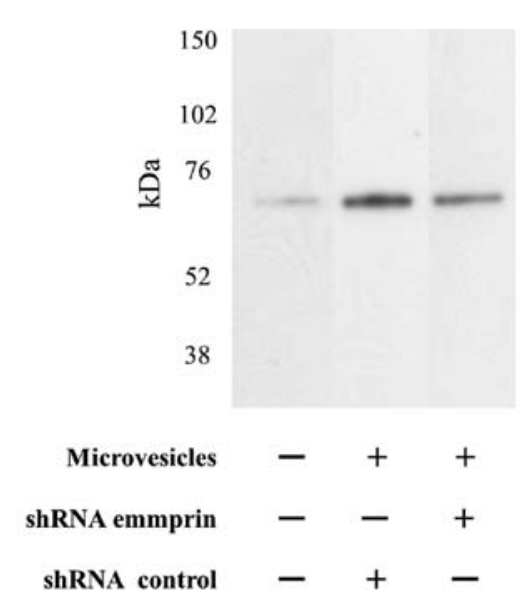

ELISA (MMP-2)

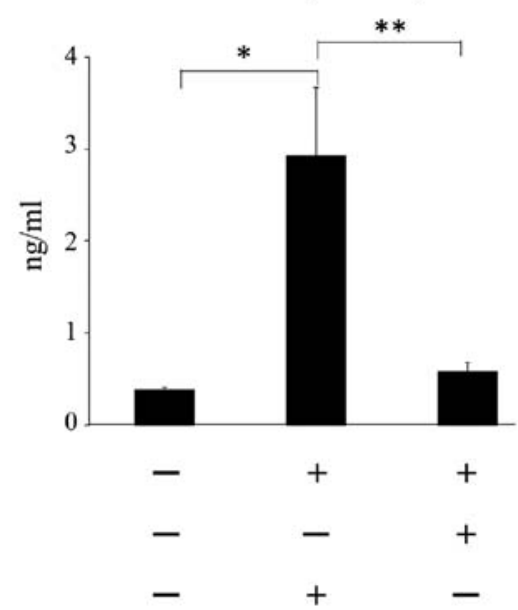

Figure 5. shRNA knockdown of emmprin in sarcoma cells and MMP-2 production from fibroblasts. (A) shRNA knockdown of emmprin also reduced emmprin expression in both total cell lysate and microvesicles. Compared to non-treatment (left lane) and control shRNA (middle lane), emmprin expression was inhibited by shRNA knockdown targeting emmprin (right lane). (B) Emmprin in microvesicles regulate MMP-2 production from fibroblasts. MMP-2 production by fibroblasts was significantly reduced when treated with microvesicles derived from sarcoma cells stably transduced with emmprin specific shRNA compared to those from control shRNA transduction. These results were confirmed by immunoblot and ELISA ( $\left.\mathrm{p}<0.01,{ }^{* *} \mathrm{p}<0.05\right)$.

regulates the production of MMP-2 from fibroblasts. siRNA or shRNA inhibition of emmprin expression in tumor cells was used to assess the role of microvesicles associated emmprin in regulation of MMP-2 production from fibroblasts (Figs. 4A and 5A). Immunoblots showed that emmprin expression was markedly reduced in both cell lysates and microvesicles derived from FU-EPS cells transfected with emmprin siRNA (Fig. 4A) or transduced with emmprin shRNA (Fig. 5A). MMP-2 production by ST353i fibroblasts was determined using ELISA upon treatment with microvesicles isolated from control siRNA transfected tumor cells or those transfected with either emmprin siRNA or shRNA. MMP-2 production from fibroblasts was increased when tumor cell microvesicles were added to the fibroblast cultures (Figs. 4B and 5B). Microvesicles collected from FU-EPS-1 transfected with emmprin-specific siRNA and transduced with emmprin-specific shRNA displayed significantly reduced MMP-2 production by fibroblasts compared with that of control cells (Figs. 4B and 5B). These findings indicate that emmprin contained in microvesicles stimulates MMP-2 production from fibroblasts.

\section{Discussion}

This is the first study to demonstrate the presence of full-length emmprin with polylactosamine in microvesicles derived from sarcoma cells and to provide evidence for the involvement of such released emmprin in the regulation of MMP-2 expression by fibroblasts.

Microvesicles, including the tumor-derived ones, are microparticles that are produced from the cell surface of plasma membrane. A recent study revealed that microvesicles shed from tumor cell lines are rather heterogeneous in size, ranging from $100 \mathrm{~nm}$ to $200 \mathrm{~nm}$, and more heterogeneous in shape than exosomes $(19,20)$. Various substances such as proteins, RNA, mRNA, and miRNA are present inside or on the surface of microvesicles. The constant secretion of microvesicles from plasma membrane results in the transport of a variety of molecules to distant sites to influence cellular processes $(10,19,21,22)$.

In the present study, we established the presence of glycosylated full-length emmprin in microvesicles derived from 
epithelioid sarcoma cells. Briefly, using multiple antibodies against emmprin (monoclonal as well as $\mathrm{N}$ - and $\mathrm{C}$-terminal polyclonal), we demonstrated that emmprin contained in microvesicles is the full-length protein identical to the form present on the plasma membrane (Fig. 1). Furthermore, the $\mathrm{N}$-glycosylation modification with the enrichment of polylactosamine was demonstrated in emmprin protein structure (Fig. 2). Previously we showed that the synthesized first immunoglobulin-like domain can mimic emmprin activity when substituted with chitobiose, the disaccharide with which $\mathrm{N}$-glycosylation starts, indicating an essential role of $\mathrm{N}$-glycosylation for the emmprin activity (16). These results support our hypothesis that biologically active emmprin is released by epithelioid sarcoma cells predominantly by microvesicle shedding, which is based on our previous report that emmprin exists in the conditioned medium as a full length protein (5).

The presence of emmprin in microvesicles was previously reported using immunoelectron microscopy in a lung carcinoma cell line (8). Expression of protein and mRNA of emmprin in microvesicles were also demonstrated in ovarian cancer and pancreatic adenocarcinoma $(10,13)$. However, there have been very few reports regarding microvesicles in sarcomas compared to those in epithelial malignant tumors. Almost 30 years ago, the presence of microvesicles was reported in a human osteogenic sarcoma cell line (23). Recently, it was reported that EWS/Fli-1 fusion mRNA of Ewing sarcoma is secreted by microvesicles (24).

Emmprin is also cleaved by MT1-MMP/MMP-14 from the cell surface and released into the culture medium $(6,25)$. Predominance between the emmprin cleaved and secreted and the emmprin shed as a vesicle is plausibly dependent upon the malignancy of the cells, and/or the difference in cell types including their aggressiveness. The variability in the abundance of microvesicles as a function of the cell type was highlighted recently. Embryonal carcinoma cells, a more aggressive testicular germ cell tumor, had a higher amount of microvesicles and a significantly higher emmprin expression level compared to seminoma cells which manifest a lesser degree of malignancy (26). In addition, it was previously reported that electron microscopy of dermatofibrosarcoma protuberans (DFSP) and dermatofibroma revealed that DFSP, the more aggressive of the two tumors, contained multivesicular buds whereas dermatofibroma lacked them (27).

It was previously reported using co-culture experiments of laryngeal cancer cells and fibroblasts that MMP-2 expression by fibroblasts requires direct cell-cell contact (28). The expression of MMP-2 by direct cell-cell contact of tumor cells and fibroblasts is undeniably established as the co-culture of tumor cells and fibroblasts clearly enhances MMP-2 expression (5). However, the upregulation of MMP-2 expression in fibroblasts in the vicinity of a tumor expressing emmprin but lacking direct contact with tumor cells, is supported by certain pathological observations $(29,30)$.

Emmprin likely exists in three cellular and extracellular forms: i) on the cell surface, ii) released after cleavage by MT1-MMP/MMP-14, and iii) secreted in the form of microvesicle shedding (31). Predominance among these forms of emmprin is likely dependent on the cell type differences including malignancy. Limitations of this study include using only one type of cells, epithelioid sarcoma, for the experi- ments. It remains to be tested that the physiological activity of emmprin in microvesicles is preserved in vivo.

In conclusion, we provided evidence that emmprin contained in microvesicles derived from sarcoma cells is involved in the regulation of MMP-2 production by fibroblasts located at sites distant from the tumor cells. We have successfully clarified a mechanism of action of emmprin, and provided insights into its important role in the invasion of sarcoma cells.

\section{Acknowledgements}

We thank M. Onitsuka, and H. Fukagawa for their excellent technical assistance.

\section{References}

1. Biswas C, Zhang Y, DeCastro R, Guo H, Nakamura T, Kataoka H and Nabeshima K: The human tumor cell-derived collagenase stimulatory factor (renamed EMMPRIN) is a member of the immunoglobulin superfamily. Cancer Res 55: 434-439, 1995.

2. Grass GD and Toole BP: How, with whom and when: An overview of CD147-mediated regulatory networks influencing matrix metalloproteinase activity. Biosci Rep 36: e00283, 2015.

3. Nakamura K, Kodama J, Hongo A and Hiramatsu Y: Role of emmprin in endometrial cancer. BMC Cancer 12: 191, 2012.

4. Sameshima T, Nabeshima K, Toole BP, Yokogami K, Okada Y, Goya T, Koono M and Wakisaka S: Glioma cell extracellular matrix metalloproteinase inducer (EMMPRIN) (CD147) stimulates production of membrane-type matrix metalloproteinases and activated gelatinase $\mathrm{A}$ in co-cultures with brain-derived fibroblasts. Cancer Lett 157: 177-184, 2000.

5. Koga K, Nabeshima K, Aoki M, Kawakami T, Hamasaki M, Toole BP, Nakayama J and Iwasaki H: Emmprin in epithelioid sarcoma: Expression in tumor cell membrane and stimulation of MMP-2 production in tumor-associated fibroblasts. Int J Cancer 120: 761-768, 2007.

6. Egawa N, Koshikawa N, Tomari T, Nabeshima K, Isobe T and Seiki M: Membrane type 1 matrix metalloproteinase (MT1-MMP/MMP-14) cleaves and releases a 22-kDa extracellular matrix metalloproteinase inducer (EMMPRIN) fragment from tumor cells. J Biol Chem 281: 37576-37585, 2006.

7. Nishio J, Iwasaki H, Nabeshima K, Ishiguro M, Naumann S, Isayama T, Naito M, Kaneko Y, Kikuchi $M$ and Bridge JA: Establishment of a new human epithelioid sarcoma cell line, FU-EPS-1: Molecular cytogenetic characterization by use of spectral karyotyping and comparative genomic hybridization. Int J Oncol 27: 361-369, 2005.

8. Sidhu SS, Mengistab AT, Tauscher AN, LaVail J and Basbaum C: The microvesicle as a vehicle for EMMPRIN in tumor-stromal interactions. Oncogene 23: 956-963, 2004.

9. Murayama T, Kataoka H, Koita H, Nabeshima K and Koono M: Glycocalyceal bodies in a human rectal carcinoma cell line and their interstitial collagenolytic activities. Virchows Arch B Cell Pathol Incl Mol Pathol 60: 263-270, 1991.

10. Baj-Krzyworzeka M, Szatanek R, Weglarczyk K, Baran J, Urbanowicz B, Brański P, Ratajczak MZ and Zembala M: Tumourderived microvesicles carry several surface determinants and mRNA of tumour cells and transfer some of these determinants to monocytes. Cancer Immunol Immunother 55: 808-818, 2006.

11. Niiya D, Egawa N, Sakamoto T, Kikkawa Y, Shinkawa T, Isobe T, Koshikawa $\mathrm{N}$ and Seiki M: Identification and characterization of Lutheran blood group glycoprotein as a new substrate of membrane-type 1 matrix metalloproteinase 1 (MT1-MMP): A systemic whole cell analysis of MT1-MMP-associating proteins in A431 cells. J Biol Chem 284: 27360-27369, 2009.

12. Koga K, Aoki M, Sameshima T, Hamasaki M, Egawa N, Seiki M, Toole BP, Suzumiya J and Nabeshima K: Synthetic emmprin peptides inhibit tumor cell-fibroblast interaction-stimulated upregulation of MMP-2 and tumor cell invasion. Int J Oncol 39: 657-664, 2011.

13. Millimaggi D, Mari M, D'Ascenzo S, Carosa E, Jannini EA, Zucker S, Carta G, Pavan A and Dolo V: Tumor vesicle-associated CD147 modulates the angiogenic capability of endothelial cells. Neoplasia 9: 349-357, 2007. 
14. Guo H, Zucker S, Gordon MK, Toole BP and Biswas C: Stimulation of matrix metalloproteinase production by recombinant extracellular matrix metalloproteinase inducer from transfected Chinese hamster ovary cells. J Biol Chem 272: 24-27, 1997.

15. Sun J and Hemler ME: Regulation of MMP-1 and MMP-2 production through CD147/extracellular matrix metalloproteinase inducer interactions. Cancer Res 61: 2276-2281, 2001.

16. Kawakami T, Sameshima T, Hojo H, Koga K, Nakahara Y, Toole BP, Suzumiya J, Okada Y, Iwasaki A and Nabeshima K: Synthetic emmprin peptides with chitobiose substitution stimulate MMP-2 production by fibroblasts. BMC Cancer 11: 300, 2011.

17. Cummings RD and Kornfeld S: Characterization of the structural determinants required for the high affinity interaction of asparagine-linked oligosaccharides with immobilized Phaseolus vulgaris leukoagglutinating and erythroagglutinating lectins. J Biol Chem 257: 11230-11234, 1982.

18. Tang W, Chang SB and Hemler ME: Links between CD147 function, glycosylation, and caveolin-1. Mol Biol Cell 15: 4043-4050, 2004

19. Cocucci E, Racchetti G and Meldolesi J: Shedding microvesicles: Artefacts no more. Trends Cell Biol 19: 43-51, 2009.

20. Muralidharan-Chari V, Clancy JW, Sedgwick A and D'Souza-Schorey C: Microvesicles: Mediators of extracellular communication during cancer progression. J Cell Sci 123 : 1603-1611, 2010.

21. Khalyfa A, Khalyfa AA, Akbarpour M, Connes P, Romana M, Lapping-Carr G, Zhang C, Andrade J and Gozal D: Extracellular microvesicle microRNAs in children with sickle cell anaemia with divergent clinical phenotypes. Br J Haematol 174: 786-798, 2016.

22. Zhang H, Bai M, Deng T, Liu R, Wang X, Qu Y, Duan J, Zhang L, Ning T, Ge S, et al: Cell-derived microvesicles mediate the delivery of miR-29a/c to suppress angiogenesis in gastric carcinoma. Cancer Lett 375: 331-339, 2016.

23. Grignani G and Jamieson GA: Tissue factor-dependent activation of platelets by cells and microvesicles of SK-OS-10 human osteogenic sarcoma cell line. Invasion Metastasis 7: 172-182, 1987.
24. Tsugita M, Yamada N, Noguchi S, Yamada K, Moritake H, Shimizu K, Akao Y and Ohno T: Ewing sarcoma cells secrete EWS/Fli-1 fusion mRNA via microvesicles. PLoS One 8: e77416, 2013.

25. Huang W, Luo WJ, Zhu P, Tang J, Yu XL, Cui HY, Wang B, Zhang Y, Jiang JL and Chen ZN: Modulation of CD147induced matrix metalloproteinase activity: Role of CD147 N-glycosylation. Biochem J 449: 437-448, 2013.

26. Milia-Argeiti E, Mourah S, Vallée B, Huet E, Karamanos NK, Theocharis AD and Menashi S: EMMPRIN/CD147-encriched membrane vesicles released from malignant human testicular germ cells increase MMP production through tumor-stroma interaction. Biochim Biophys Acta 1840: 2581-2588, 2014.

27. Dominguez-Malagon H, Valdez-Carrillo MC and CanoValdez AM: Dermatofibroma and dermatofibrosarcoma protuberans: A comparative ultrastructural study. Ultrastruct Pathol 30: 283-291, 2006.

28. Suzuki S, Sato M, Senoo H and Ishikawa K: Direct cell-cell interaction enhances pro-MMP-2 production and activation in co-culture of laryngeal cancer cells and fibroblasts: Involvement of EMMPRIN and MT1-MMP. Exp Cell Res 293: 259-266, 2004.

29. Kanekura T, Chen X and Kanzaki T: Basigin (CD147) is expressed on melanoma cells and induces tumor cell invasion by stimulating production of matrix metalloproteinases by fibroblasts. Int J Cancer 99: 520-528, 2002.

30. Nabeshima K, Suzumiya J, Nagano M, Ohshima K, Toole BP, Tamura K, Iwasaki H and Kikuchi M: Emmprin, a cell surface inducer of matrix metalloproteinases (MMPs), is expressed in T-cell lymphomas. J Pathol 202: 341-351, 2004.

31. Nabeshima K, Iwasaki H, Koga K, Hojo H, Suzumiya J and Kikuchi M: Emmprin (basigin/CD147): Matrix metalloproteinase modulator and multifunctional cell recognition molecule that plays a critical role in cancer progression. Pathol Int 56: 359-367, 2006. 